\title{
Analysis of Caesarean Section Rates Using the Robson Classification System at a University Hospital in Spain
}

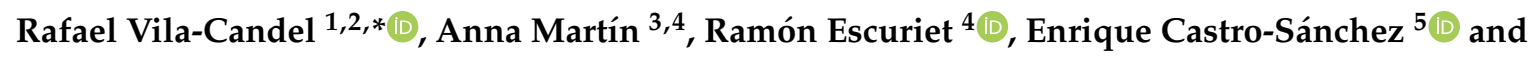 \\ Francisco Javier Soriano-Vidal ${ }^{2,6}$ (1) \\ 1 Department of Obstetrics and Gynaecology, Hospital Universitario de la Ribera, FISABIO. Crta. Corbera km \\ 1, 46600 Valencia, Spain \\ 2 Department of Nursing, Faculty of Nursing and Podiatry, Universitat de València, Jaume Roig, s/n, \\ 46010 Valencia, Spain; soriano_fravid@gva.es \\ 3 School of Medicine, Universidad Autónoma de Madrid, Spain. C/Arzobispo Morcillo 4, 28029 Madrid, \\ Spain; annama7@blanquerna.url.edu \\ 4 School of Health Sciences Blanquerna, Universitat Ramon Llull, C/Padilla 326, 08025 Barcelona, Spain; \\ rescuriet@gencat.cat \\ 5 NIHR Health Protection Research Unit (HPRU) in Healthcare Associated Infections (HCAI) and \\ Antimicrobial Resistance (AMR) at Imperial College London, Du Cane Road, London W12 0NN, UK; \\ e.castro-sanchez@imperial.ac.uk \\ 6 Department of Obstetrics and Gynaecology, Hospital Lluis Alcanyis, FISABIO. Crta Xàtiva, s/n, 46800 \\ Valencia, Spain \\ * Correspondence: vila_rafcan@gva.es
}

Received: 8 January 2020; Accepted: 26 February 2020; Published: 29 February 2020

\begin{abstract}
Background: The WHO recommends the use of the Robson ten-group classification system (RTGCS) as an effective monitoring and analysis tool to assess the use of caesarean sections (CS). The present study aimed to conduct an analysis of births using the RTGCS in La Ribera University Hospital over nine years and to assess the levels and trends of CS births. Methods: Retrospective study between January 1, 2010, and December 31, 2018. All eligible women were allocated in RTGCS to determine the absolute and relative contribution made by each group to the overall CS rate; linear regression and weighted least squares regression analysis were used to analyze trends over time. The risk of CS of women with induced versus spontaneous onset of labor was calculated with an odds ratio (OR) with a $95 \%$ CI. Results: 16,506 women gave birth during the study period, $19 \%$ of them by CS. Overall, $20.4 \%$ of women were in group 1 (nulliparous, singleton cephalic, term, spontaneous labor), $29.4 \%$ in group 2 (nulliparous, singleton cephalic, term, induced labor or caesarean before labor), and $12.8 \%$ in group 4 (multiparous, singleton cephalic, term, induced or caesarean delivery before labor) made the most significant contributions to the overall rate of CS; Conclusions: In our study, Robson Groups 1, 2, and 4, were identified as the main contributors to the hospital's overall CS rate. The RTGCS provides an easy way of collecting information about the CS rate, is a valuable clinical method that allows standardized comparison of data, and time point, and identifies the groups driving changes in CS rates.
\end{abstract}

Keywords: caesarean section; Robson ten-group classification system; labor; delivery classification; Spain

\section{Background}

There is growing international concern about the increased use of caesarean sections (CS), particularly in high-income countries [1]. Caesarean procedures performed in the absence of a clinical 
justification do not reduce maternal or infant death rates if carried out at a rate higher than $10 \%-15 \%$ [2]. The unjustified, excessive use of clinical procedures can lead to an ever-increasing therapeutic cascade of avoidable interventions [3] and become life-threatening in the present or future pregnancies for both the women and children [4]. The worldwide rise in CS rates has become a growing public health concern and a cause for debate due to potential maternal and perinatal risks, cost issues, and inequity in access [5].

There is a high degree of variability in the reported crude rates of CS performed in different countries and regions, and there are often even significant differences between hospitals within a single region. The highest caesarean rates are observed in the Dominican Republic (56.4\%), Brazil (55.6\%), and Egypt (51.8\%), with Africa (7.3\%) showing the lowest proportion of these procedures [1]. In most European countries, the rates are about $25 \%$ to $35 \%$ [5]. In Spain, the average CS rate reported across the 17 autonomous communities, the governing entities independently responsible for health care [6] and for deploying health resources to serve the needs of their local populations, was found to be $24.5 \%$ in $2015[7,8]$. However, due to the decentralized structure of the health system, there is no nationally established system to monitor the use of caesarean procedures.

Achieving reductions in maternal and infant morbidity and mortality are, among others, the objectives promoted by the World Health Organization (WHO) for 2030. One of the suggested ways to meet this goal consists of avoiding clinically unnecessary caesareans [9]. However, the challenge is to keep CS rates low while ensuring safe outcomes for mothers and infants [4]. One of the main referred difficulties was the lack of a classification tool that would be feasible to be used internationally, to allow audit feedback and setting an optimal CS rate over countries. To address this gap, in 2001, Robson et al. proposed an overall classification method that facilitates an understanding of the rate of CS in a center and makes it possible to identify key subpopulation groups, all in order to inform measures aimed at preventing unnecessary procedures [10-12].

The WHO has proposed the use of the Robson ten-group classification system (RTGCS) as the global standard, as this classification method allows for the analysis of changing trends over time, makes it possible to compare differences between centers and shed light on how changes in clinical practice can optimize caesarean rates, thus ensuring excellence in maternal and perinatal care [13]. The more in-depth analysis offered by this method allows us to examine issues such as which groups of women and which obstetric populations are most likely to undergo caesarean sections, information that can point us toward the kinds of interventions that might help reduce the rates of caesarean sections, when and where such reductions are desirable [14].

The present study aimed to conduct an analysis of births using the RTGCS in La Ribera University Hospital over nine years and to assess the levels and trends of CS births.

\section{Methods}

\subsection{Design, Population, and Sample}

An observational study was conducted of births at La Ribera University Hospital (LRUH) (Valencia, Spain) from January 1, 2010, to December 31, 2018. The data were retrospectively extracted from the electronic birth records of women included over this period.

The health department of La Ribera has a population of 250,000 inhabitants and a yearly average of 1700 births. The hospital manages births from week 34, as below this gestational age, women are referred when appropriate to the reference hospital for neonatal unit support if required. The study's population included women giving birth during the study period to live babies after at least 24 weeks gestation, or to a liveborn baby weighing at least $500 \mathrm{~g}$.

Our study applied the ethical principles for medical research established in current Spanish legislation and was approved by the LRUH Research Commission's Research Ethics Committee (\#134-19). Considerations such as confidentiality and full information were extended to all participants. 


\subsection{Data Collection Tools}

We used the RTGCS to categorize all women giving birth with 24 weeks' gestation or longer during our study period [15]. Table 1 shows the definitions of each group.

Table 1. Group description of Robson's classification system.

\begin{tabular}{|l|}
\hline Nulliparous, singleton cephalic, $\geq 37$ weeks, spontaneous labor. \\
2a Nulliparous, singleton cephalic, $\geq 37$ weeks, induced labor. \\
2b Nulliparous, singleton cephalic, $\geq 37$ weeks, or caesarean delivery before labor. \\
3 Multiparous, singleton cephalic, $\geq 37$ weeks, spontaneous labor. \\
4a Multiparous, singleton cephalic, $\geq 37$ weeks, induced labor. \\
4b Multiparous, singleton cephalic, $\geq 37$ weeks, caesarean delivery before labor. \\
5 Previous caesarean delivery, singleton cephalic, $\geq 37$ weeks, spontaneous labor or induced labor or caesarean delivery \\
before labor. \\
6 All nulliparous singleton breeches, spontaneous labor or induced labor or caesarean delivery before labor. \\
7 All multiparous singleton breeches (including previous caesarean delivery), spontaneous labor or induced labor or \\
caesarean delivery before labor. \\
8 All multiple pregnancies, spontaneous labor or induced labor or caesarean delivery before labor. \\
9 All abnormal singleton lies (including previous caesarean delivery but excluding breech), spontaneous labor or \\
induced labor or caesarean delivery before labor. \\
10 All singleton cephalic, $\leq 36$ weeks (including previous caesarean delivery), spontaneous labor or induced labor or \\
caesarean delivery before labor.
\end{tabular}

All women were contemporaneously classified using the five obstetric characteristics described in the RTGCS (number of fetuses, parity, fetal presentation, the onset of labor, and gestational age), without requiring the indication for CS (Figure 1). All categories were totally inclusive and mutually exclusive.

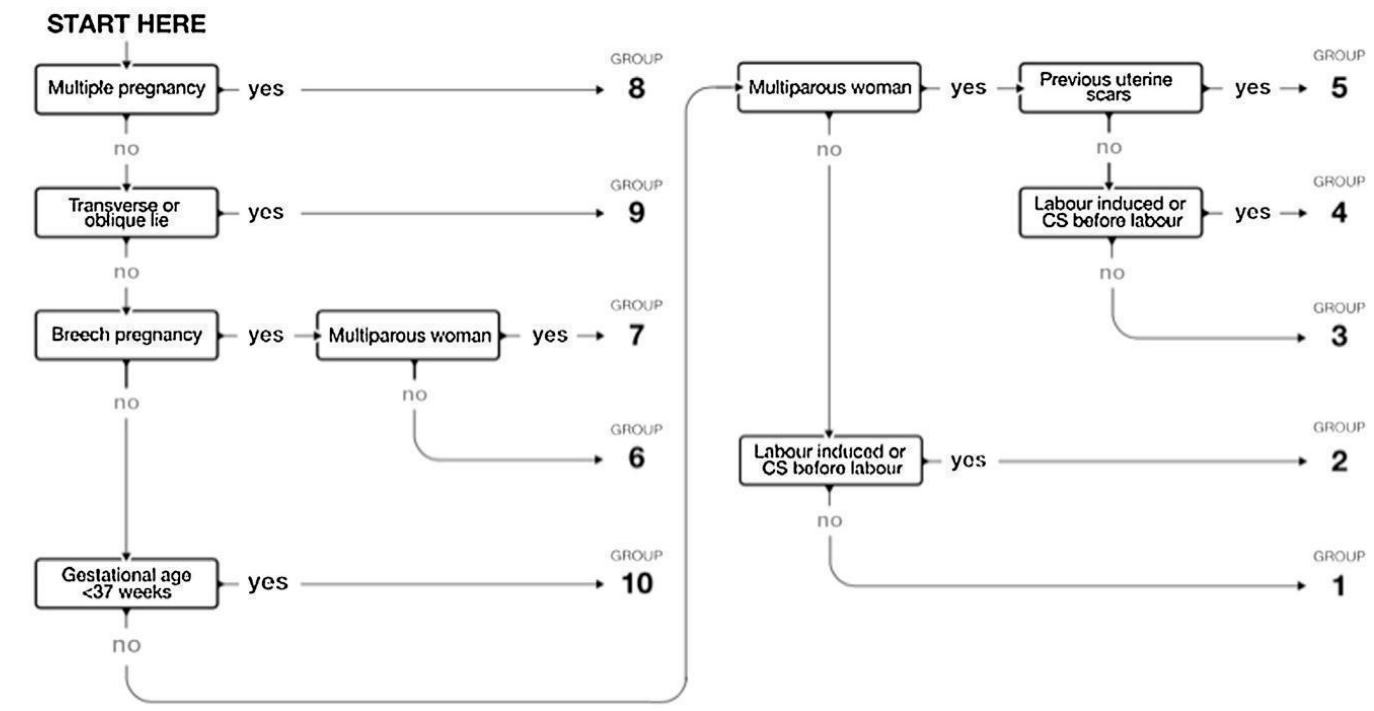

Figure 1. Flow chart for the Robson ten-group classification system (RTGCS). Source https://www.who. int/reproductivehealth/publications/maternal_perinatal_health/robson-classification/en/ [15].

A training session was conducted to introduce the implementation manual to the staff responsible for data collection. The interpretation of the data collected in the report table of the Robson classification was carried out according to three main domains: quality of information, type of population, and caesarean section rate.

The five variables collected for RTGCS included obstetric history (parity and previous caesarean section), type onset of labor (spontaneous, induced, or caesarean section before the onset of labor), fetal presentation or lie (cephalic, breech, or transverse), number of neonates, and gestational age (preterm or term). Other information collected to describe population consisted of sociodemographic data and the obstetric characteristics of the pregnant women: country of origin, age, newborn's gender, birth weight, and feeding in the delivery room (breastfeeding, formula feeding). 


\subsection{Statistical Analysis}

Statistical analyses were carried out using SPSS software version 20.0 (IBM Corp. Released 2011. IBM SPSS Statistics for Windows, Armonk, NY: IBM Corp.) Frequencies and percentages were calculated for all variables. The standard deviation $(x \pm S D)$ of the mean was calculated for quantitative variables. The overall CS rate, the relative size of each group, the CS rate within each group, and each group's relative and absolute contribution to the overall CS rate were calculated over the study period. The relative size of each of the 10 groups was calculated by dividing the number of births in each group by the total number of births in the obstetric population and expressing it as a percentage. The CS rates were calculated by dividing the number of CS by the total number of births in each group and expressing this figure as a percentage. Finally, the percentage contribution made by each group to the overall CS rate was calculated by dividing the number of CS in each group by the total number of births in the obstetric population.

Linear regression was performed to determine the trend over time in the number of deliveries. Weighted least squares (WLS) regression was used to analyze the trends in CS over time, weighted for the total number in each ten-croup classification system group in that year. The Chi-square test was used to analyze the statistical significance of the differences in numbers of CS between the different groups. In order to calculate the risk of CS of women with induced versus spontaneous onset of labor (groups 2 and 4 vs. groups 1 and 3), an odds ratio (OR) with a 95\% CI, was calculated. The significance level was set at $p<0.05$.

\section{Results}

In terms of the characteristics of the population, the women's mean age was $30.7 \pm 5.6$ years; $54.4 \%(8977 / 16506)$ were nulliparous, and $81.9 \%$ (13521/16506) were natives of Spain. Of the newborns, $51.4 \%(8097 / 15764)$ were males. The mean birth weight of the infants was $3273 \pm 518 \mathrm{~g}$. In terms of the feeding method employed after childbirth, $73.3 \%$ (12100/15988) of women chose to breastfeed their infants, with nulliparous women displaying a higher rate in this regard (78.1\% [6792/8687]; $p<0.001)$.

The total number of births over the nine years covered by this study was 16,506, and the rate of caesarean sections performed over the period was 19\% (Table 2). There was an increase in the CS rate from $18.4 \%$ in 2010 to $20.8 \%$ in 2018 by $0.8 \%(95 \%$ CI $-0.79-0.86)$ annually $(p<0.001)$. There was a decrease in the total number of births over the time period (mean difference between 2010/2018: -279; 95\% CI 265-292; $p<0.001)$.

Table 2. Distribution of the study's population according to Robson's ten-group classification system, relative and overall contribution to the CS rate $(n=16,506)$.

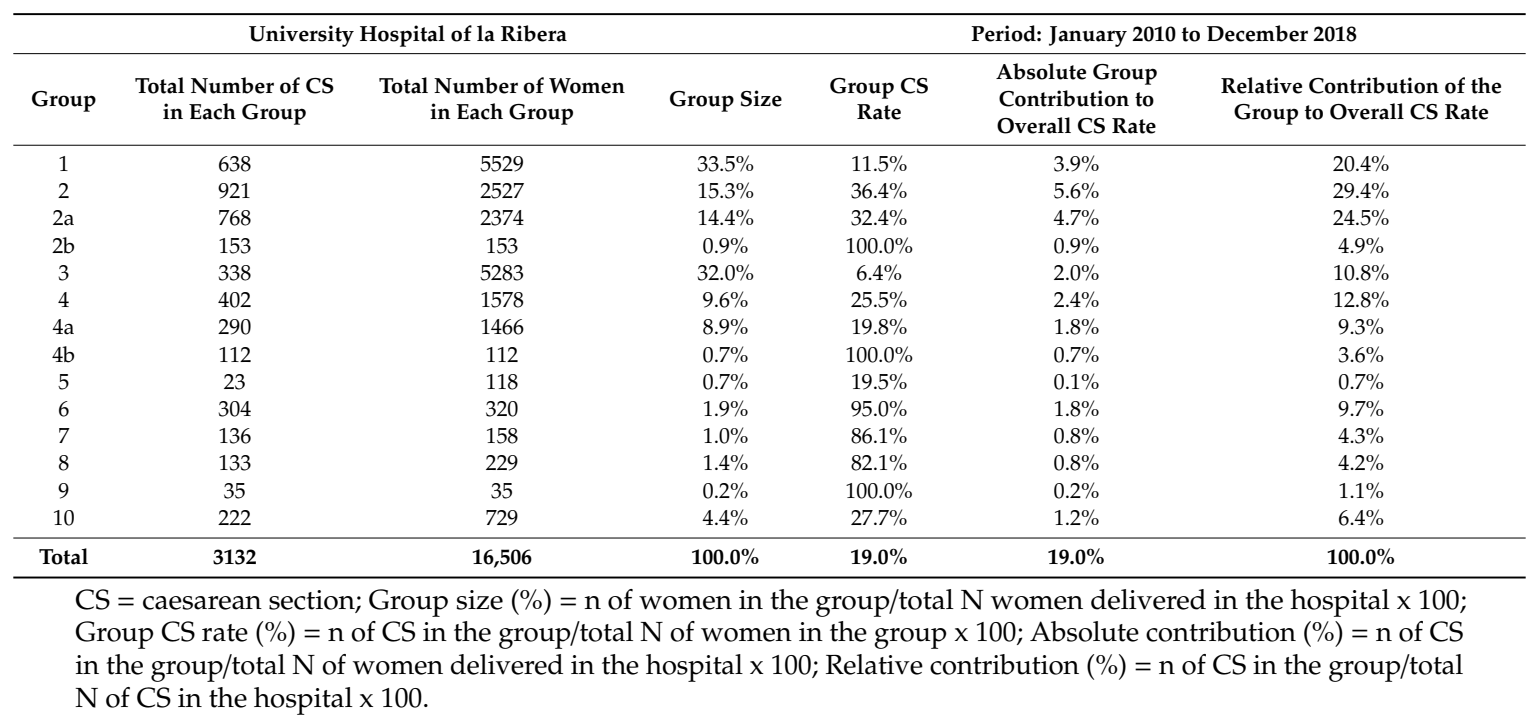


Table 2 presents the distribution of the study's population in the RTGCS and their relative and overall contribution to the CS rate. Figure 2 shows the absolute contribution of each group to the overall CS rate over time.

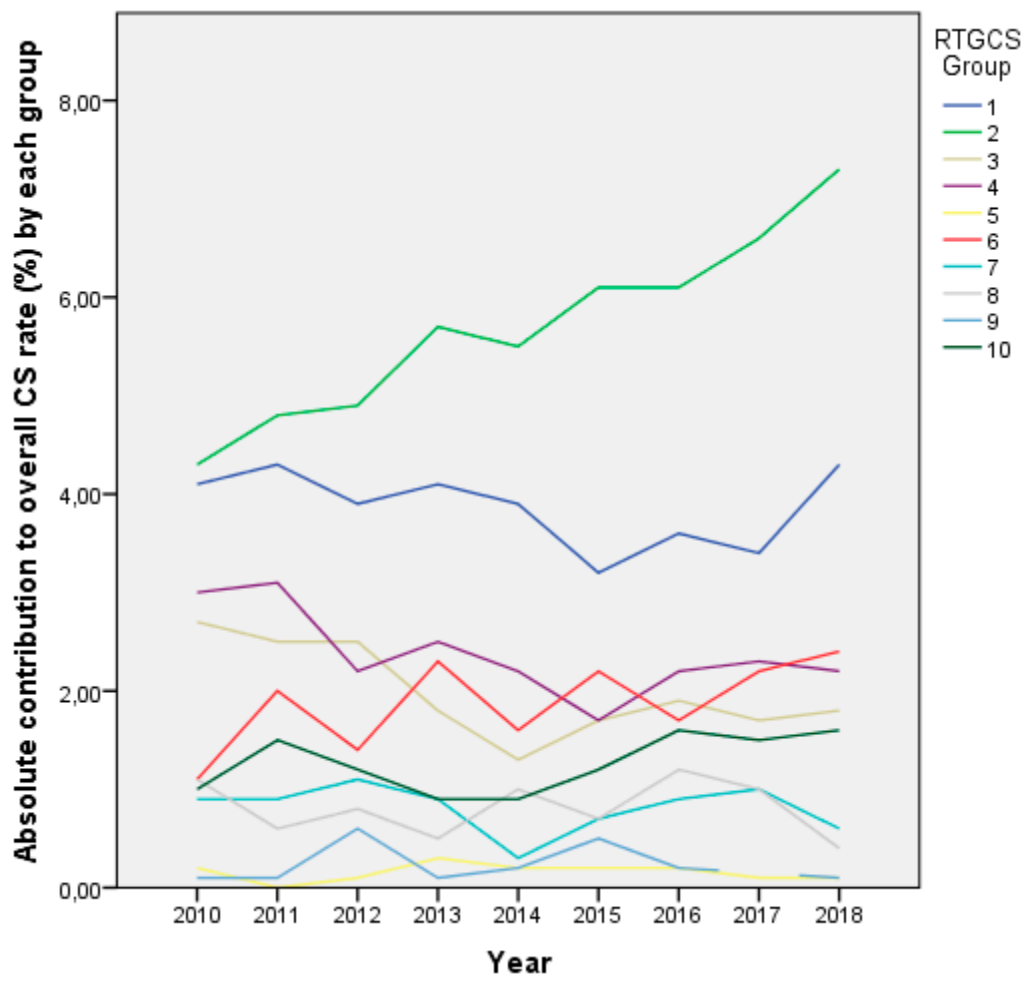

Figure 2. Absolute contribution to the overall caesarean section (CS) rate of each group from 2010 to 2018. RTGCS $=$ Robson ten-group classification system.

Table 3 demonstrates the trends in the proportions of women in the ten groups over time. The data show that nulliparous women, singleton cephalic, term, classified in group 1 (spontaneous labor) and 2 (induced labor or caesarean before labor) represented $48.9 \%$ of the total sample, whereas multiparous women with singleton pregnancies who had not undergone a previous CS (groups 3 and 4 ) were $41.6 \%$. The relative contribution of group 1 to the global CS rate decreased, from $22.1 \%$ in 2010 to $20.5 \%$ in 2018 (reduction of $0.56 \%$ per year). On the other hand, it increased in group 2, going from $23.2 \%$ in 2010 to $34.9 \%$ in 2018 (an increase of $1.50 \%$ per year).

Table 3. Trends in the proportions of women in the RTGCS over time (weighted least squares regression).

\begin{tabular}{cccc}
\hline Group & Change in Overall \% Per Year & $\mathbf{9 5 \%}$ CI & $p$-Value \\
\hline 1 & -0.56 & $-0.58,-0.55$ & 0.001 \\
2 & 1.50 & $1.48-1.53$ & 0.001 \\
3 & -0.72 & $-0.74,-0.71$ & 0.001 \\
4 & -0.69 & $-0.71,-0.66$ & 0.001 \\
5 & -0.81 & $-1.13,-0.50$ & 0.001 \\
6 & 0.12 & $0.11-0.22$ & 0.031 \\
7 & -0.19 & $-0.25,-0.13$ & 0.001 \\
8 & -0.24 & $-0.31,-0.17$ & 0.001 \\
9 & 0.87 & $0.24-0.97$ & 0.269 \\
10 & 0.24 & $0.21-0.27$ & 0.001 \\
\hline
\end{tabular}

CI: confidence interval. 
The most significant contribution to the overall total number of CS performed came from the women placed in group 2. We observed that group 1 was 2.2 times larger than group 2, meaning that the number of cases of spontaneous initiation of labor was higher than those of induced labor or elective caesareans among the nulliparous. In 2010, the ratio between groups 1 and 2 was 2.7:1 and in 2018, it was 1.7:1.

The relative contribution to the overall CS rate of groups 3 and 4 has reduced over the years. Group 3 has gone from $14.5 \%$ in 2010 to $8.9 \%$ in 2018 (reduction of $0.72 \%$ per year), and group 4 has gone from $16.6 \%$ in 2010 to $10.6 \%$ in 2018 (reduction of $0.69 \%$ per year). The comparison between groups 3 (multiparous, singleton cephalic, term, spontaneous labor) and 4 (multiparous, singleton cephalic, term, induced or caesarean delivery before labor) yielded a difference of an even greater magnitude, as the size of group 3 was 3.3 times that of group 4 . The ratio between the sizes of groups 3 and 4 increased from 3.1: 1 in 2010 to 3.4: 1 in 2018.

The relative contribution of group 5, vaginal birth after a caesarean (VBAC) to the global CS rate decreased from $0.9 \%$ in 2010 to $0.7 \%$ in 2018 (reduction $0.81 \%$ per year).

The relative contribution of group 6 (all nulliparous women with a single breech pregnancy) to the global CS rate increased, from $6.0 \%$ in 2010 to $11.6 \%$ in 2018 - an increase of $0.12 \%$ per year. In contrast, group 7 (all multiparous women with a single breech pregnancy including women with previous uterine scars) reduced, from $5.1 \%$ in 2010 to $2.7 \%$ in 2018 -a reduction of $0.19 \%$ per year. The ratio of the size of group 6 to that of group 7 was 2.0, indicating that breech presentations were more frequent in nulliparous than in multiparous women. As overall, the ratio between groups 6 and 7 increased from 1.1: 1 in 2010 to 4.4: 1 in 2018.

The relative contribution of group 8 (all multiple pregnancies) to the global CS rate has decreased, from $5.7 \%$ in 2010 to $1.7 \%$ in 2018 - a reduction of $0.24 \%$ per year. The relative contribution of group 10 to the global CS rate increased significantly, from $5.5 \%$ in 2010 to $7.5 \%$ in 2018 - an increase of $0.24 \%$ per year.

We were interested in analyzing the differences between the onset of labor (spontaneous/induced) and the mode of birth (vaginal/caesarean section) in groups 1 to 4 (Figure 3). Group 1 vs. 2: 638/4891 CS from spontaneous onset of labor vs. 768/1606 CS from induced onset of labor; Group 3 vs. 4: 338/4945 CS from spontaneous onset labor vs. 290/1176 CS from induced onset of labor. We observed that the induction of labor triples the risk of having a caesarean section delivery, compared to the onset of spontaneous labor. The risk was for both, in the primiparous (group 2 vs. 1: OR $=3.6$; $95 \%$ CI 3.2-4.1; $p<0.001$ ) as well as in the multiparous group (group 4 vs. 3: $\mathrm{OR}=3.6 ; 95 \% \mathrm{CI} 3.0-4.3 ; p<0.001$ ).

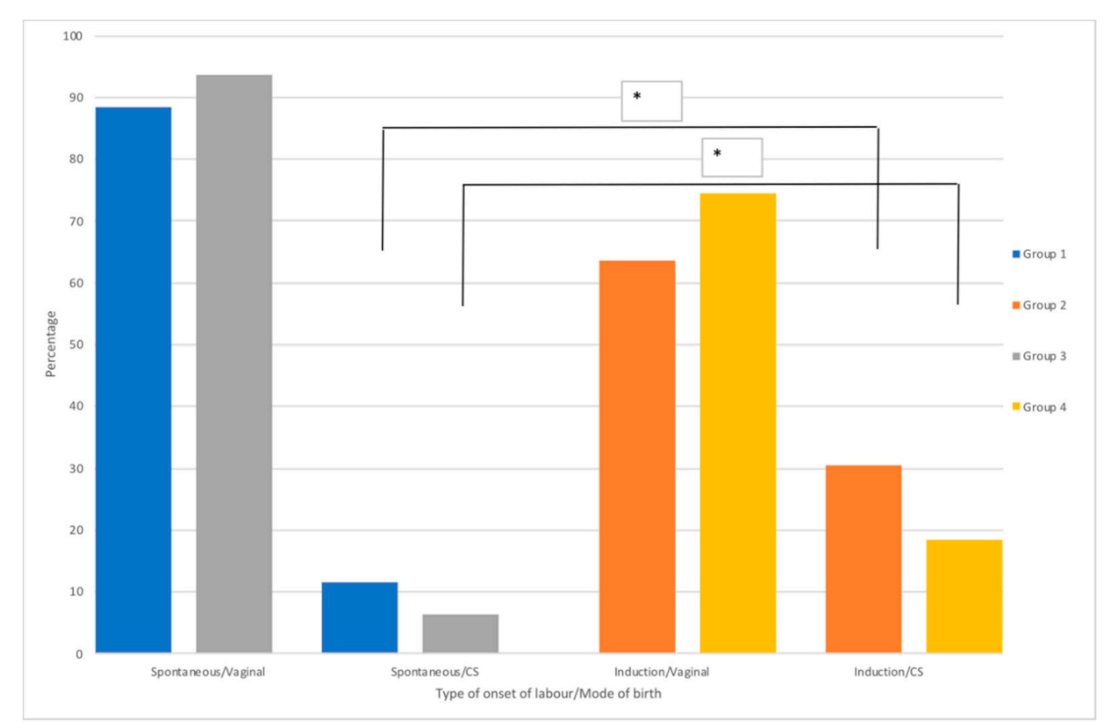

Figure 3. Distribution of type onset of labor by mode of birth between groups 1 vs. 3 , and 2 vs. 4 in the study period. Odds ratio analyses $\left({ }^{*} p<0.001\right)$. 
We analyzed the indication for induction of labor in each of the groups ( $2 a$ and $4 a$ ) and their relation to the mode of birth as shown in Table 4. We observed that the first cause of induction was prolonged pregnancy (PP), with a total caesarean rate of $21.1 \%$ in these induced labors, being higher in group 2. In the case of pregnancy-induced hypertension, oligoamnios and prenatal anomalies on the cardiotocographic (CTG) fetal monitoring, there was an increased rate of CS and the analysis showed differences between groups.

Table 4. Distribution of indication for induction of labor in groups ( $2 a$ and $4 a$ ) and their relation to the mode of birth $(n=3840)$, Chi-square analyses.

\begin{tabular}{|c|c|c|c|c|c|c|c|c|c|c|c|}
\hline \multirow{4}{*}{$\begin{array}{l}\text { Induction of } \\
\text { Labor Indication }\end{array}$} & & & \multicolumn{9}{|c|}{ Group $(n=3840)$} \\
\hline & & & \multicolumn{4}{|c|}{$2 a(n=2374)$} & \multicolumn{4}{|c|}{$4 a(n=1466)$} & \multirow[b]{3}{*}{$p$-Value } \\
\hline & & & \multicolumn{2}{|c|}{ CS $(n=768)$} & \multicolumn{2}{|c|}{ Vaginal $(n=1606)$} & \multicolumn{2}{|c|}{ CS $(n=290)$} & \multicolumn{2}{|c|}{ Vaginal $(n=1176)$} & \\
\hline & n & $\%$ Total Row & $\mathbf{n}$ & $\%$ col & $\mathrm{n}$ & $\%$ col & $\mathbf{n}$ & $\%$ col & n & $\%$ col & \\
\hline Anomalies on the CTG & 193 & $5.0 \%$ & 63 & $8.2 \%$ & 54 & $3.4 \%$ & 23 & $7.9 \%$ & 53 & $4.5 \%$ & 0.001 \\
\hline Prolonged pregnancy & 812 & $21.1 \%$ & 177 & $23.0 \%$ & 341 & $21.2 \%$ & 57 & $19.7 \%$ & 237 & $20.2 \%$ & $<0.001$ \\
\hline Polyhydramnios & 72 & $1.9 \%$ & 17 & $2.2 \%$ & 19 & $1.2 \%$ & 10 & $3.4 \%$ & 26 & $2.2 \%$ & 0.088 \\
\hline $\begin{array}{l}\text { Pregnancy-induced } \\
\text { hypertension }\end{array}$ & 189 & $4.9 \%$ & 68 & $8.8 \%$ & 66 & $4.1 \%$ & 16 & $5.5 \%$ & 39 & $3.3 \%$ & 0.007 \\
\hline $\begin{array}{c}\text { Antepartum hemorrhage in } \\
\text { the 3rd trimester }\end{array}$ & 16 & $0.4 \%$ & 2 & $0.3 \%$ & 12 & $0.7 \%$ & 1 & $0.3 \%$ & 1 & $0.1 \%$ & 0.226 \\
\hline Not recorded & 303 & $7.9 \%$ & 26 & $3.4 \%$ & 133 & $8.3 \%$ & 13 & $4.5 \%$ & 131 & $11.1 \%$ & 0.057 \\
\hline Oligohydramnios & 305 & $7.9 \%$ & 69 & $9.0 \%$ & 130 & $8.1 \%$ & 19 & $6.6 \%$ & 87 & $7.4 \%$ & 0.002 \\
\hline Fetal pathology & 4 & $0.1 \%$ & 3 & $0.4 \%$ & 0 & $0,0 \%$ & 0 & $0.0 \%$ & 1 & $0.1 \%$ & 0.046 \\
\hline Maternal pathology & 52 & $1.4 \%$ & 6 & $0.8 \%$ & 20 & $1.2 \%$ & 4 & $1.4 \%$ & 22 & $1.9 \%$ & 0.482 \\
\hline Anhydramnios & 22 & $0.6 \%$ & 0 & $0,0 \%$ & 15 & $0.9 \%$ & 0 & $0.0 \%$ & 7 & $0.6 \%$ & - \\
\hline Favorable cervix & 145 & $3.8 \%$ & 14 & $1.8 \%$ & 57 & $3.5 \%$ & 8 & $2.8 \%$ & 66 & $5.6 \%$ & 0.135 \\
\hline Small for gestational age & 113 & $2.9 \%$ & 21 & $2.7 \%$ & 59 & $3.7 \%$ & 7 & $2.4 \%$ & 26 & $2.2 \%$ & 0.573 \\
\hline Latent phase & 14 & $0.4 \%$ & 3 & $0.4 \%$ & 5 & $0.3 \%$ & 1 & $0.3 \%$ & 5 & $0.4 \%$ & 0.393 \\
\hline PROM not described & 355 & $9.2 \%$ & 55 & $7.2 \%$ & 164 & $10.2 \%$ & 28 & $9.7 \%$ & 108 & $9.2 \%$ & 0.327 \\
\hline $\mathrm{PROM}<12 \mathrm{H}$ & 14 & $0.4 \%$ & 2 & $0.3 \%$ & 6 & $0.4 \%$ & 0 & $0.0 \%$ & 6 & $0.5 \%$ & 0.186 \\
\hline $\mathrm{PROM}>12 \mathrm{H}<18 \mathrm{H}$ & 127 & $3.3 \%$ & 23 & $3.0 \%$ & 46 & $2.9 \%$ & 8 & $2.8 \%$ & 50 & $4.3 \%$ & 0.011 \\
\hline $\mathrm{PROM}>18 \mathrm{H}<24 \mathrm{H}$ & 131 & $3.4 \%$ & 20 & $2.6 \%$ & 62 & $3.9 \%$ & 9 & $3.1 \%$ & 40 & $3.4 \%$ & 0.422 \\
\hline $\mathrm{PROM}>24 \mathrm{H}$ & 390 & $10.2 \%$ & 83 & $10.8 \%$ & 178 & $11.1 \%$ & 31 & $10.7 \%$ & 98 & $8.3 \%$ & 0.112 \\
\hline Meconium stained liquor & 272 & $7.1 \%$ & 48 & $6.3 \%$ & 110 & $6.8 \%$ & 24 & $8.3 \%$ & 90 & $7.7 \%$ & 0.085 \\
\hline Suspected macrosomia & 62 & $1.6 \%$ & 10 & $1.3 \%$ & 22 & $1.4 \%$ & 7 & $2.4 \%$ & 23 & $2.0 \%$ & 0.485 \\
\hline Intrauterine growth restriction & 153 & $4.0 \%$ & 34 & $4.4 \%$ & 69 & $4.3 \%$ & 15 & $5.2 \%$ & 35 & $3.0 \%$ & 0.708 \\
\hline Gestational diabetes & 79 & $2.1 \%$ & 21 & $2.7 \%$ & 30 & $1.9 \%$ & 7 & $2.4 \%$ & 21 & $1.8 \%$ & 0.150 \\
\hline Abnormal dopplers & 17 & $0.4 \%$ & 3 & $0.4 \%$ & 8 & $0.5 \%$ & 2 & $0.7 \%$ & 4 & $0.3 \%$ & 0.793 \\
\hline
\end{tabular}

CS: caesarean section; CTG: cardiotocographic fetal monitoring; PROM: prolonged rupture of membranes.

\section{Discussion}

The present study includes 16,506 births that were attended at the Ribera Hospital during the nine years of the study period. When studying the evolution of the activity during this period of time, we observed an annual reduction of attended births. By contrast, there is an annual increase in the global CS rate, which has meant an increase. The data show that there has been a clear trend towards increased use of CS over this period, a finding that echoes those of other studies conducted in Spain $[8,14]$ and is aligned with worldwide trends, although the rate recorded is lower than the Spanish national average and that of other European countries [13,16]. The common overuse of CSs is a significant public health concern, and of considerable debate, due to potential maternal and perinatal risks that raise health care costs, childbirth admissions, and inequity in the access to maternity health care $[17,18]$.

Several previous studies document a significant association between advanced maternal age ( $>35$ years) and an increased likelihood of CS birth $[19,20]$. This association may be interpreted as a result of a changing social environment, but a common explanation is the pre-pregnancy morbidities associated in these cases [21]. However, as noted in Table 4, only a few women that constitute our study presented comorbidity and, none of them had the age reported, as was not required for the RTGCS. From our data, then, we would not be able to provide a hypothesis to the relation between maternal age and the risk of a CS birth.

The nulliparous population included in groups 1 and 2 (nulliparous, singleton cephalic, term), was the most significant contributor to the overall CS rate. This increase is consistent with previous studies and probably shows a link to negative consequences in women's health in high-income 
countries [3,22-24]. The relative contribution of group 1 (spontaneous labor) to the overall CS rate decreased significantly over the study period, in line with other studies [25]. Group 2 (induced labor) makes the greatest absolute contribution to the overall CS rate ( $4.7 \%$ overtime period), and this is as per previously published [26]. The CS rate for this group increased significantly over the study period from $23.2 \%$ in 2010 to $34.9 \%$ in 2018 . These rates of CS are similar to those that have been previously reported in studies examining CS rates in European countries using the RTGCS [22,27]. Previous reports have described that a ratio of less than 2:1 between the sizes of groups 1 and 2 may reflect a high incidence of induction and CS before labor [11]. In 2010, this ratio was 2.7:1 and in 2018, 1.7:1. Furthermore, during the analyzed period, the CS rate increased in group 2, thereby contributing significantly to the overall increased CS rate.

The relative contribution to the overall CS rate from groups 3 and 4 (multiparous, singleton cephalic, term) has been reduced over the years, as observed in other studies [27]. Even though the relative contribution of group 4a (induced multiparous women) to the global CS rate has also decreased significantly, it ranks as the third group in terms of relative contribution to the global CS rate in the study. The group sizes for groups 3 and 4 has increased, from 3.1: 1 in 2010 to 3.4: 1 in 2018, and this might explain the decrease in the number of inductions in multiparous women. In addition, there was an increase in the number of multiparous women with spontaneous onset of labor, being able to explain the decrease in the number of caesarean sections in group 4a during the study.

Once the main contributors to CS rates are identified, the next steps should be focusing on potential interventions to prevent the further $\mathrm{CS}$ rise. The single most common indication for induction in groups 2a and 4a was PP and, together with all types of PROM except meconium-stained liquor, they represent almost half of all indications for induction of labor. We also noticed that, in $16.3 \%$ of the medical records, the cause for an induction was not stated. In addition, in our results and as per other studies [28], we observed that induction of labor, both in nulliparous and multiparous women, increases the risk of having a caesarean section. Until the 2018 release of a large trial regarding labor induction versus expectant management [29], evidence suggested that induction of labor without medical indication was associated with an increased rate of caesarean birth [17]. In order to reduce the number of unnecessary caesarean sections, it is very relevant to assess the medical need for induction of labor appropriately and document this promptly [24].

In contrast with other studies, we observe that the contribution of group 5 (women with singleton cephalic full-term pregnancy, who have undergone at least one caesarean section) to the overall CS rate was smaller than in other countries like France [23], UK [30], and Canada [31], and has been decreasing during the study period. However, it was greater than Ireland [27], Norway, or Sweden [30]. Furthermore, our hospital has adopted new guidelines for clinical practice that recommend offering to women, who meet optimal clinical conditions with a singleton pregnancy of cephalic presentation at $37+0$ weeks or beyond and who have had a single previous lower segment caesarean delivery, the option of having a vaginal birth [32]. This new policy might have contributed to a significant decrease in the rates of caesarean sections among the members of this group. Furthermore, significant differences between countries in VBAC rates may suggest different obstetrical care practices, some of them facilitating the increase on VBAC rates.

Lastly, groups 8 (multiple pregnancies) and 10 (premature births) have an expected contribution of the general CS rate similar to that reported by Robson and other authors $[4,11,23,31,33,34]$.

There is a need to analyze the possible causes of the global steady growth that has been observed in the overall rate of caesarean sections. To this end, it is important to seek out classification systems that will allow us to make comparisons between different health care systems [1,35]. An earlier systematic review comparing different classification methods concluded that the Robson classification is optimal for monitoring CS [1], and the WHO has recommended adopting the Robson classification as the global standard tool for monitoring CS [4]. The application of the Robson model is a critical step in the efforts to optimize the use of these procedures, as it helps identify, analyze, and shed light on how these interventions are employed among specific, relevant groups at a given institution. It is 
documented that the RTGCS is a valuable clinical method that allows standardized comparations of data across countries and can be used as a common starting point to audit induction of labor and caesarean deliveries routinely $[13,22,36]$. Furthermore, the use of the classification system applied in this study across Spanish hospitals would help to bring awareness on each hospital performance, facilitate the comparison between hospitals and regions and align the maternity services with the current evidence and every hospital specific need. After this study was conducted, regular audits and feedback using the Robson classification system were implemented in our hospital in order to identify issues with existing practice to improve the overall quality of care.

It is essential to avoid unnecessary interventions in childbearing women, and at the same time, ensure that those interventions that are necessary take place [37]. Every effort must be made to perform these procedures on the women that truly need them rather than merely attempting to reach a given optimal rate [13]. With this perspective in mind, it is even more important to apply suitable methods to monitor and assess the results of these kinds of interventions in order to identify when and where they are overused, mainly when they are performed on healthy women who are not deemed to be at risk. The maternity team at the hospital, including the obstetric and midwifery team [17], studied here, conducts a daily, in-depth review of every caesarean section performed on the previous day in order to assess whether the clinical indications followed met the standards set out in the institution's protocols and to provide feedback to the healthcare professional involved.

Due to the complexity of the different interconnected factors that influence the rising CS rates, interventions aimed to reduce unnecessary CS have only shown moderate success to date [1]. Any increases in obesity, age, and nulliparity among populations of women are not enough to explain increases. Addressing the non-medical reasons that drive caesarean sections, therefore, is key to reducing inappropriate use [38]. In accordance to Vogel et al. [22], factors associated with higher rates of vaginal births may include firm policies on CS due to maternal request, cultural or social pressure, differences in the legal framework for medical litigation, and strategies favoring home births, midwifery-led continuity models of care and approach to birth $[39,40]$. High-quality research is needed in the future to evaluate multicomponent and locally tailored interventions addressing women's and health professionals' demands as well as the health system when attempting to design and implement interventions aiming at reducing the number of unnecessary CS [41].

\section{Strengths and Limitations}

The strengths of the study include the fact that few studies in Spain have analyzed the caesarean rate in a single facility. Moreover, the sample was collected rigorously, and a sample size that was large and sufficient for the estimations made. It, therefore, provides a valuable addition to the existing evidence as it provides a successful application of the Robson classification to analyze the CS rate in a setting like a tertiary hospital and the results could be compared with other hospitals or regions. Besides, the results obtained using the Robson classification method confirm the quality of the data collected under the guidelines set out by the method's author for this purpose.

Among the limitations of this study is the possible existence of recording errors in medical records. Any errors that were detected were analyzed by the research team and recoded to reflect the data on the obstetric process collected in the medical record.

\section{Conclusions}

In our study, the main contributors to the overall CSs performed came from Robson groups 1, 2, and 4. Efforts to reduce the overall CS rate must focus on reducing the initial CS rate (groups 1 and 2). Conducting a review of the indications for inducing labor might be one of the keys to achieving a decrease in the number of caesarean sections performed at this institution. The worldwide increase in the rate of caesarean section over the past few decades has made evident the need to formulate and apply a classification system (such as the 10-group Robson method) that makes possible a comparison of the caesarean rates at different hospitals. Such a system can be used to identify the groups displaying 
the most significant growth in the frequency of these procedures so as to act to stem these increases and provide an easy way of collecting information about CS rate.

Author Contributions: R.V.-C. designed the protocol and led the project; R.V.-C. collected the necessary data; R.V.-C. and F.J.S.-V. performed the statistical analysis. All authors contributed to the interpretation of results. R.V.-C., A.M., E.C.-S. and R.E. wrote the first draft, with all authors providing critical comments. All authors have read and agreed to the published version of the manuscript.

Funding: This project has been funded by the Conselleria de Educación, Investigación, Cultura y Deporte of the Generalitat Valenciana in its call for grants for the conduction of R\&D\&I projects developed by emerging research groups in 2018 (Reference GV/2018/036).

Acknowledgments: We are grateful to all the staff at the hospital and health facilities of University Hospital La Ribera for their support with this research. E.C.-S. is affiliated to the National Institute for Health Research (NIHR) Health Protection Research Unit (NIHR HPRU) [HPRU-2012-10047] in Healthcare Associated Infection and Antimicrobial Resistance at Imperial College London in partnership with Public Health England (PHE) and is a NIHR Senior Nurse and Midwife Research Leader. E.C.-S. has received a Welcome ISSF Faculty postdoctoral fellowship, an Early Career Research Fellowship from the Antimicrobial Research Collaborative at Imperial College London and acknowledges the support of the Florence Nightingale Foundation and the NIHR Imperial Patient Safety Translational Research Centre. The views expressed in this article are those of the authors and not necessarily those of the NIHR, or the Department of Health and Social Care.

Conflicts of Interest: The authors declare no conflict of interest.

\section{Abbreviations}

$\begin{array}{ll}\text { CTG } & \text { Cardiotocographic fetal monitoring } \\ \text { CS } & \text { Caesarean Section } \\ \text { PP } & \text { Prolonged pregnancy } \\ \text { PROM } & \text { Prolonged Rupture of Membranes } \\ \text { RTGCS } & \text { Robson ten-group classification system } \\ \text { LRUH } & \text { La Ribera University Hospital } \\ \text { VBAC } & \text { Vaginal Birth After Caesarean } \\ \text { WHO } & \text { World Health Organization }\end{array}$

\section{References}

1. Betrán, A.P.; Ye, J.; Moller, A.B.; Zhang, J.; Gülmezoglu, A.M.; Torloni, M.R. The increasing trend in caesarean section rates: Global, regional and national estimates: 1990-2014. PLoS ONE 2016, 11, e0148343. [CrossRef] [PubMed]

2. Cagan, M.; Tanacan, A.; Aydin Hakli, D.; Beksac, M.S. Changing rates of the modes of delivery over the decades $(1976,1986,1996,2006$, and 2016) based on the Robson-10 group classification system in a single tertiary health care center. J. Matern.-Fetal Neonatal. Med. 2019, 7058, 1-8. [CrossRef] [PubMed]

3. Souza, J.P.; Gülmezoglu, A.M.; Lumbiganon, P.; Laopaiboon, M.; Carroli, G.; Fawole, B.; Ruyan, P. Caesarean section without medical indications is associated with an increased risk of adverse short-term maternal outcomes: The 2004-2008 WHO Global Survey on Maternal and Perinatal Health. BMC Med. 2010, 8, 71. [CrossRef] [PubMed]

4. Tura, A.K.; Pijpers, O.; De Man, M.; Cleveringa, M.; Koopmans, I.; Gure, T.; Stekelenburg, J. Analysis of caesarean sections using Robson 10-group classification system in a university hospital in eastern Ethiopia: A cross-sectional study. BMJ Open 2018, 8, e020520. [CrossRef]

5. Kirchengast, S.; Hartmann, B. Recent lifestyle parameters are associated with increasing caesarean section rates among singleton term births in Austria. Int. J. Environ. Res. Public Health 2019, 16, 14. [CrossRef]

6. Rodríguez-Blanco, N.; Tuells, J.; Vila-Candel, R.; Nolasco, A. Adherence and concordance of influenza and pertussis vaccination coverage in pregnant women in Spain. Int. J. Environ. Res. Public Health 2019, 16, 543. [CrossRef]

7. OECD. Health at a Glance 2017: OECD Indicators; OECD Publishing: Paris, France, 2017; pp. 180-181.

8. Aguilar Redondo, R.; Manrique Fuentes, G.; Aisa Denaroso, L.M.; Delgado Martínez, L.; González Acosta, V.; Aceituno Velasco, L. Uso de la clasificación de Robson en un Hospital Comarcal de España para reducir la tasa de cesáreas. Rev. Chil. Obstet. Ginecol. 2016, 81, 99-104. [CrossRef] 
9. Ye, J.; Zhang, J.; Mikolajczyk, R.; Torloni, M.R.; Gülmezoglu, A.M.; Betran, A.P. Association between rates of caesarean section and maternal and neonatal mortality in the 21st century: A worldwide population-based ecological study with longitudinal data. BJOG Int. J. Obstet. Gynaecol. 2016, 123, 745-753. [CrossRef]

10. Betrán, A.P.; Vindevoghel, N.; Souza, J.P.; Gülmezoglu, A.M.; Torloni, M.R. A systematic review of the Robson classification for caesarean section: What works, doesn't work and how to improve it. PLoS ONE 2014, 9, e97769. [CrossRef]

11. Robson, M. The ten group classification system (TGCS)-a common starting point for more detailed analysis. BJOG Int. J. Obstet. Gynaecol. 2015, 122, 701. [CrossRef]

12. Chen, I.; Opiyo, N.; Tavender, E.; Mortazhejri, S.; Rader, T.; Petkovic, J.; Yogasingam, S.; Taljaard, M.; Agarwal, S.; Laopaiboon, M.; et al. Non-clinical interventions for reducing unnecessary caesarean section. Cochrane Database Syst. Rev. 2018, CS005528. [CrossRef] [PubMed]

13. Betran, A.P.; Torloni, M.R.; Zhang, J.J.; Gülmezoglu, A.M. WHO statement on caesarean section rates. BJOG Int. J. Obstet. Gynaecol. 2016, 123, 667-670. [CrossRef] [PubMed]

14. Cabeza Vengoechea, P.J.; Calvo Pérez, A.; Betrán, A.P.; del Mar Morey, M.M.; Febles Borges, M.M.; Alcácer, P.X.; Alcover, A.R.; Campos Villamiel, E.F.; Morán Antolín, E.M.; Rehkugler, S.; et al. Clasificación de cesáreas por Grupos de Robson en dos periodos comparativos en el Hospital de Manacor. Progresos Obstet. Ginecol. 2010, 53, 385-390. [CrossRef]

15. WHO. Robson Classification: Implementation Manual; WHO: Geneva, Switzerland, 2017; ISBN 978-92-4-151319-7.

16. WHO; HRP. WHO Statement on Caesarean Section Rates; WHO: Geneva, Switzerland, 2015; pp. 667-670.

17. Smith, D.C.; Phillippi, J.C.; Lowe, N.K.; Breman, R.B.; Carlson, N.S.; Neal, J.L.; Gutierrez, E.; Tilden, E.L. Using the Robson 10-Group Classification System to Compare Cesarean Birth Utilization Between US Centers With and Without Midwives. J. Midwifery Women Health 2019, 65, 10-21. [CrossRef]

18. Tapia, V.; Betran, A.P.; Gonzales, G.F. Caesarean section in Peru: Analysis of trends using the Robson classification system. PLoS ONE 2016, 11, e0148138. [CrossRef]

19. Dunn, L.; Kumar, S.; Beckmann, M. Maternal age is a risk factor for caesarean section following induction of labor. Aust. N. Z. J. Obstet. Gynaecol. 2017, 57, 426-431. [CrossRef]

20. Yoshioka-Maeda, K.; Ota, E.; Ganchimeg, T.; Kuroda, M.; Mori, R. Caesarean section by maternal age group among singleton deliveries and primiparous Japanese women: A secondary analysis of the WHO Global Survey on Maternal and Perinatal Health. BMC Pregnancy Childbirth 2016, 16, 39. [CrossRef]

21. Rydahl, E.; Declercq, E.; Juhl, M.; Maimburg, R.D. Cesarean section on a rise-Does advanced maternal age explain the increase? A population register-based study. PLoS ONE 2019, 14. [CrossRef]

22. Vogel, J.P.; Betrán, A.P.; Vindevoghel, N.; Souza, J.P.; Torloni, M.R.; Zhang, J.; Tunçalp, Ö.; Mori, R.; Morisaki, N.; Ortiz-Panozo, E.; et al. Use of the robson classification to assess caesarean section trends in 21 countries: A secondary analysis of two WHO multicountry surveys. Lancet Glob. Health 2015, 5, e260-e270. [CrossRef]

23. Lafitte, A.S.; Dolley, P.; Le Coutour, X.; Benoist, G.; Prime, L.; Thibon, P.; Dreyfus, M. Rate of caesarean sections according to the Robson classification: Analysis in a French perinatal network-Interest and limitations of the French medico-administrative data (PMSI). J. Gynecol. Obstet. Hum. Reprod. 2018, 47, 39-44. [CrossRef]

24. Assar, T.M.; Nucier, A.A.A.R.; Raziq, H.E.A.; Abd El-Azym Saad, A.S.; Megahed Amer, W.; Jadoon, B. Analysis of the caesarean section rate using the 10-Group Robson classification at Benha University Hospital, Egypt. Women Birth 2019, 33, e105-e110. [CrossRef]

25. Macfarlane, A.J.; Blondel, B.; Mohangoo, A.D.; Cuttini, M.; Nijhuis, J.; Novak, Z.; Ólafsdóttir, H.S.; Zeitlin, J. Wide differences in mode of delivery within Europe: Risk-stratified analyses of aggregated routine data from the Euro-Peristat study. BJOG Int. J. Obstet. Gynaecol. 2016, 123, 559-568. [CrossRef] [PubMed]

26. Kacerauskiene, J.; Bartuseviciene, E.; Railaite, D.R.; Minkauskiene, M.; Bartusevicius, A.; Kliucinskas, M.; Simoliuniene, R.; Nadisauskiene, R.J. Implementation of the Robson classification in clinical practice: Lithuania's experience. BMC Pregnancy Childbirth 2017, 17, 432. [CrossRef] [PubMed]

27. Crosby, D.A.; Murphy, M.M.; Segurado, R.; Byrne, F.; Mahony, R.; Robson, M.; McAuliffe, F.M. Cesarean delivery rates using Robson classification system in Ireland: What can we learn? Eur. J. Obstet. Gynecol. Reprod. Biol. 2019, 236, 121-126. [CrossRef]

28. Rydahl, E.; Eriksen, L.; Juhl, M. Effects of induction of labor prior to post-term in low-risk pregnancies: A systematic review. JBI Database Syst. Rev. Implement. Rep. 2019, 17, 170-208. [CrossRef] 
29. Grobman, W.A.; Rice, M.M.; Reddy, U.M.; Tita, A.T.N.; Silver, R.M.; Mallett, G.; Hill, K.; Thom, E.A.; El-Sayed, Y.Y.; Perez-Delboy, A.; et al. Labor induction versus expectant management in low-risk nulliparous women. N. Engl. J. Med. 2018, 379, 513-523. [CrossRef]

30. Brennan, D.J.; Robson, M.S.; Murphy, M.; O'Herlihy, C. Comparative analysis of international cesarean delivery rates using 10-group classification identifies significant variation in spontaneous labor. Am. J. Obstet. Gynecol. 2009, 201, 308-e1-308.e8. [CrossRef]

31. Roberge, S.; Dubé, E.; Blouin, S.; Chaillet, N. Reporting Caesarean Delivery in Quebec Using the Robson Classification System. J. Obstet. Gynaecol. Canada 2017, 39, 152-156. [CrossRef]

32. RCOG Birth After Previous Caesarean Birth. Available online: https://www.rcog.org.uk/globalassets/ documents/guidelines/gtg_45.pdf (accessed on 16 March 2019).

33. Hehir, M.P.; Ananth, C.V.; Siddiq, Z.; Flood, K.; Friedman, A.M.; D'Alton, M.E. Cesarean delivery in the United States 2005 through 2014: A population-based analysis using the Robson 10-Group Classification System. Am. J. Obstet. Gynecol. 2018, 219, 105-e1-105.e11. [CrossRef]

34. Zeitlin, J.; Szamotulska, K.; Drewniak, N.; Mohangoo, A.D.; Chalmers, J.; Sakkeus, L.; Irgens, L.; Gatt, M.; Gissler, M.; Blondel, B. Preterm birth time trends in Europe: A study of 19 countries. BJOG Int. J. Obstet. Gynaecol. 2013, 120, 1356-1365. [CrossRef]

35. Robson, M.S. Classification of caesarean sections. Fetal Matern. Med. Rev. 2001, 12, 23-39. [CrossRef]

36. Souza, J.P.; Betran, A.P.; Dumont, A.; De Mucio, B.; Gibbs Pickens, C.M.; Deneux-Tharaux, C.; Ortiz-Panozo, E.; Sullivan, E.; Ota, E.; Togoobaatar, G.; et al. A global reference for caesarean section rates (C-Model): A multicountry cross-sectional study. BJOG Int. J. Obstet. Gynaecol. 2016, 123, 427-436. [CrossRef] [PubMed]

37. Seijmonsbergen-Schermers, A.; De Jonge, A.; Van Den Akker, T.; Beeckman, K.; Bogaerts, A.; Barros, M.; Janssen, P.; Binfa, L.; Rydahl, E.; Frith, L.; et al. Variations in childbirth interventions in high-income countries: Protocol for a multinational cross-sectional study. BMJ Open 2018, 8, e017993. [CrossRef]

38. The Lancet Stemming the global caesarean section epidemic. Lancet 2018, 392, 1279. [CrossRef]

39. Renfrew, M.J.; McFadden, A.; Bastos, M.H.; Campbell, J.; Channon, A.A.; Cheung, N.F.; Silva, D.R.A.D.; Downe, S.; Kennedy, H.P.; Malata, A.; et al. Midwifery and quality care: Findings from a new evidence-informed framework for maternal and newborn care. Lancet 2014, 384, 1129-1145. [CrossRef]

40. Sandall, J.; Soltani, H.; Gates, S.; Shennan, A.; Devane, D. Midwife-led continuity models versus other models of care for childbearing women. Cochrane Database Syst. Rev. 2016, 4, CD004667. [CrossRef]

41. Betrán, A.P.; Temmerman, M.; Kingdon, C.; Mohiddin, A.; Opiyo, N.; Torloni, M.R.; Zhang, J.; Musana, O.; Wanyonyi, S.Z.; Gülmezoglu, A.M.; et al. Interventions to reduce unnecessary caesarean sections in healthy women and babies. Lancet 2018, 392, 1358-1368. [CrossRef]

(C) 2020 by the authors. Licensee MDPI, Basel, Switzerland. This article is an open access article distributed under the terms and conditions of the Creative Commons Attribution (CC BY) license (http://creativecommons.org/licenses/by/4.0/). 